\title{
Clinical status and detection of periodontopathogens and Streptococcus mutans in children with high levels of supragingival biofilm
}

\section{Sheila Cavalca Cortelli(a) José Roberto Cortelli(a) Davi Romero Aquino(b) Marinella Holzhausen ${ }^{(a)}$ Gilson Cesar Nobre Franco(c) Fernando de Oliveira Costa ${ }^{(\mathrm{d})}$ Daniel Fine ${ }^{(e)}$}

\footnotetext{
(a) PhD, Professor; (b)PhD student - Department of Periodontology, University of Taubaté, SP, Brazil.

(c) PhD, Professor, Department of Oral Biology, University of Taubaté, SP, Brazil.

(d) $\mathrm{PhD}$, Professor, Department of

Periodontology, Federal University of Minas Gerais, MG, Brazil.

(e) $\mathrm{PhD}$, Professor, Department of Oral Biology, New Jersey Dental School, USA.
}

\begin{abstract}
Knowledge about the presence of some important oral pathogens is an important step in better identifying children at risk for periodontal and/or caries diseases in later life. The purpose of this study was to detect the presence of Streptococcus mutans (Sm), Aggregatibacter actinomycetemcomitans (Aa), Campylobacter rectus (Cr), Porphyromonas gingivalis (Pg), Prevotella intermedia (Pi), and Tannerella forsythia (Tf) in gingival biofilm samples from 196 children, and to assess whether any of these pathogens are more associated with gingival inflammation extension and the Decayed/Missing/Filled teeth (DMFT/dmft) index. The subjects presented plaque index greater than $80 \%$ and were divided in 3 groups according to the bleeding index (BI): I) Low bleeding ( $\leq 30 \%)$, II) Medium bleeding ( $31-59 \%)$ and III) High bleeding ( $\geq 60 \%)$. The presence of each pathogen was determined by PCR. The prevalence of Sm was $71.9 \%$ and the mean $\mathrm{dmft} / \mathrm{DMFT}$ was 6.68 . The prevalence in low, medium and high bleeding groups was $43.5 \%, 34.5 \%$ and $46.7 \%$ for Aa; $43.5 \%, 37.9 \%$, and $36.7 \%$ for $\mathrm{Cr}$; $99.1 \%, 100 \%$, and $96.7 \%$ for Pg; $56.5 \%, 56.9 \%$, and $66.7 \%$ for $\mathrm{Pi}$; and $58.3 \%, 60.3 \%$, and $56.7 \%$ for Tf, respectively. $\mathrm{Pg}(99.0 \%)$ was the most prevalent periodontal pathogen detected followed by Tf (58.7\%), Pi (58.2\%), Aa (41.3\%) and Cr (40.8\%). Our study indicated that in this high plaque index population studied, a high prevalence of Sm and high mean DMFT were observed. In addition, the presence of Pi was associated with the presence of inflammation $(\mathrm{P}<0.05)$ whereas $\mathrm{Cr}$ was associated with periodontal health $(\mathrm{P}<0.05)$.
\end{abstract}

Descriptors: Periodontal diseases; Dental caries; Dental plaque; Diagnosis.
Corresponding author:

Sheila Cavalca Cortelli

Secretaria de Pós-Graduação em

Odontologia - UNITAU

Rua Expedicionário Ernesto Pereira, 110 ,

Centro - Taubaté - SP - Brazil

CEP: 12020-330

E-mail:cavalcacortelli@uol.com.br

Received for publication on May 14, 2008

Accepted for publication on Jan 15, 2009 


\section{Introduction}

Periodontal diseases are infections in which specific bacteria play an important role. The continuous dental plaque accumulation may result in an imbalance between pathogenic species and the host defense mechanisms, which may lead to gingival inflammation. ${ }^{1}$ In fact, poor oral hygiene status has frequently been associated with a high prevalence or severity of periodontal disease..$^{2-4}$

Current knowledge clearly shows that the microbiota associated with healthy periodontal sites is quite different from that of inflamed sites. Increased frequency of detection of Aggregatibacter actinomycetemcomitans (Aa), Porphyromonas gingivalis (Pg), Tannerela forsythia (Tf), Treponema denticola (Td), Prevotella intermedia (Pi), Fusobacterium nucleatum (Fn), Campylobacter rectus (Cr), and Eikenella corrodens (Ec) has been found in periodontal lesions. ${ }^{5}$

On the other hand, the bacterium Streptococcus mutans $(\mathrm{Sm})$ is generally accepted to be the principal etiological agent of dental caries due to its high cariogenic potential. ${ }^{6}$ Accordingly, higher levels of $S$. mutans have been associated with a higher risk for dental caries. ${ }^{7}$ Thus, microbiological diagnosis of some of these specific pathogens should improve our ability to identify individuals or sites at risk for developing oral diseases.

Therefore, it is reasonable to believe that knowledge about the colonization process that takes place early in life is an important step in better identifying which children need more effective oral treatments in order to minimize the risk of periodontal and/or caries diseases in later life. In view of the controversial data available on the early infection by oral pathogens, the aim of the present study was to detect the presence of selected oral pathogens, using PCR, in gingival marginal biofilm samples from Brazilian children with high levels of supragingival biofilm, and to assess whether any of these pathogens are more associated with gingival inflammation extension and DMFT index.

\section{Materials and Methods Subject population}

This study was conducted in accordance with guidelines of the 196/96 resolution of the National Health Council (NHC) and was approved by the University of Taubaté Ethics Committee (protocol \# 0076/01). The children's parents or guardians received complete information regarding the objectives and procedures of the study and provided written informed consent.

One hundred and ninety six school children with mixed dentition (92 male and 104 female), ranging in age from 6 to 12 years $(8.6 \pm 1.4$ years), from a public school in Taubaté (SP, Brazil) were enrolled in this cross-sectional study. The children in need of dental care were referred to the Dental Clinics of the University of Taubaté for appropriate treatment.

The children presented good general health and had not received treatment with antibiotics within the past 6 months prior to the study. In addition, the children selected for this experiment should present a high plaque index (minimum value of $80 \%$ for the Plaque Assessment Scoring System - $\mathrm{PASS}^{8}$ ).

Patients presenting systemic diseases, immunodeficiency and/or use of orthodontic or prosthetic devices were excluded from this study.

\section{Clinical examination and microbial sampling}

The clinical exam and microbial samples collection were performed in the children by two calibrated examiners. Intra- and inter-examiner agreement was high (minimum kappa value was 0.83 ).

Clinical examination comprised the determination of dental biofilm by PASS. ${ }^{8}$ A total of 208 children were initially examined in this study. According to the cut-off point, plaque index $>80 \%$, 12 children were excluded; therefore, 196 children (92 male and 104 female) completed the study. The bleeding index $(\mathrm{BI})^{9}$ was also evaluated. Dental status was assessed by the number of decayed, missing, filled teeth (dmft/DMFT), based on World Health Organization criteria (WHO). ${ }^{10}$ For BI and dmft/ DMFT, both dental arches were examined in their entirety. In accordance with BI, the children were divided into 3 groups: I) Low bleeding ( $\leq 30 \%$ ), II) Medium bleeding (31\% - 59\%) and III) High bleeding $(\geq 60 \%)$.

A pooled gingival marginal biofilm sample was 
obtained from the mesial aspect of the same 5 PASS index teeth, i.e, first molars and right upper incisor. In absence of first molars or right upper incisor, second molars or left superior incisor were sampled. Before the collection procedure, cotton rolls were applied to prevent contamination of the sampling area with other oral fluids. The supragingival biofilm was gently removed with sterile cotton pellets and gingival marginal biofilm samples were collected using sterile paper points (Tanari \#30, Tanariman Industrial Ltda., Manacapuru, AM, Brazil) inserted to the depth of the gingival sulci during 60 seconds. This procedure was done for each of the five sites previously selected and the paper points of each subject were placed in a same microtube with $1 \mathrm{ml}$ of reduced Ringer's solution $(0.9 \mathrm{~g}$ sodium chloride, $0.042 \mathrm{~g}$ potassium chloride, $0.025 \mathrm{~g}$ calcium chloride, $100 \mathrm{ml}$ distilled water) on ice. The samples were stored at $-80^{\circ} \mathrm{C}$ until the analyses.

\section{Microbial analysis by Polymerase Chain Reaction - PCR}

The bacterial cells in the microtube were dispersed using a Vortex ${ }^{\mathrm{TM}}$ and centrifuged for $3 \mathrm{~min}$ at $12,000 \mathrm{rpm}$. The genomic DNA was extracted from the bacterial pellet using a DNA purification Kit (InstaGene ${ }^{\mathrm{TM}}$ purification matrix, BioRad Laboratories, Hercules, CA, USA) according to the manufacturer's instructions.

The polymerase chain reaction (PCR) method was performed according to the protocol described by Cortelli et al. ${ }^{11}$ (2008), using specific primers for Streptococcus mutans ${ }^{12}$, Aggregatibacter actinomycetemcomitans, ${ }^{13}$ Porphyromonas gingivalis, ${ }^{13}$ Prevotella intermedia, ${ }^{13}$ Campylobacter rectus ${ }^{13}$ and Tannerella forsythia. ${ }^{13}$

\section{Statistical analysis}

Analysis of variance (ANOVA) was used for comparisons of the number of children in the different groups (Low, Medium and High bleeding). Student's $t$ post hoc test was applied when appropriate. The prevalence of periodontopathogens among the groups was analyzed by the Chi-squared test $\left(\mathrm{X}^{2}\right)$. The Kruskal-Wallis test was used to compare $S$. $m u$ tans and DMFT index.

Finally, Pearson's test was used for the assessment of possible associations between $S$. mutans and the other periodontal pathogens ( $\mathrm{Aa}, \mathrm{Cr}, \mathrm{Pg}, \mathrm{Pi}$ and $\mathrm{T} f$ ). Statistical significance was established at $5 \%$.

\section{Results}

Based on the Bleeding index (BI), the children were divided into three groups (Table 1). And, among children with high levels of supragingival biofilm we found a higher number of children with low bleeding scores $(p<0.05)$.

The microbial analysis by PCR indicated that 1 child $(0.5 \%)$ was negative for all periodontal bacteria studied; 41 children $(20.9 \%)$ were positive for one species, 42 children (21.2\%) were positive for two species, 36 children $(18.7 \%)$ were positive for three species, 29 children $(14.8 \%)$ were positive for four species and 47 children $(24.0 \%)$ were positive for all bacteria studied. The most prevalent periodontopathogen was $P$. gingivalis $(99.0 \%)$, followed by T. forsythia $(58.7 \%)$, P. intermedia $(58.2 \%)$, A. actinomycetemcomitans $(41.3 \%)$ and C. rectus $(40.8 \%)$. Finally, the overall prevalence of $S$. mutans was $71.9 \%$.

The analysis of the prevalence of each bacterium among the different groups indicated that no statistically significant difference $(p>0.05)$ was observed in the detection frequencies of $S$. mutans $(71.3 \%$, $77.6 \%$ and $63.3 \%$ for Low, Medium and High bleeding, respectively), P. gingivalis $(99.1 \%, 100 \%$ and 96.7\% for Low, Medium and High bleeding, respectively) and T. forsythia $(58.3 \%, 60.3 \%$ and $56.7 \%$

\begin{tabular}{|c|c|c|c|c|}
\hline \multirow{4}{*}{$\begin{array}{r}\text { Table } 1 \text { - Demographic } \\
\text { characteristics of the study } \\
\text { volunteers }(n=196) \text {. }\end{array}$} & & Low Bleeding ( $\leq 30 \%)$ & Medium Bleeding (31-59\%) & High Bleeding ( $\geq 60 \%$ ) \\
\hline & $N$ & 108 children* & 58 children & 30 children \\
\hline & Gender & 56 male / 52 female & 24 male / 34 female & 12 male / 18 female \\
\hline & Age & $8.7 \pm 1.4$ years & $8.6 \pm 1.2$ years & $8.5 \pm 1.5$ years \\
\hline
\end{tabular}


for Low, Medium and High bleeding, respectively).

We also showed that a higher prevalence of $P$. intermedia was associated with gingival inflammation extension, since its detection frequency in the High Bleeding group $(66.7 \%)$ was higher than in the Low $(56.5 \%)$ and Medium (57.6\%) bleeding groups. C. rectus was associated with periodontal health, because its detection frequency in the Low bleeding group $(43.5 \%)$ was higher than in the Medium $(37.9 \%)$ and High bleeding groups (36.7\%). No statistical difference was observed between Low and Medium bleeding $(p>0.05)$.

The detection frequencies for all bacteria studied are shown in Graph 1. A. actinomycetemcomitans was more prevalent in the Low $(43.5 \%)$ and High bleeding $(46.7 \%)$ groups when compared to the Medium bleeding group $(34.5 \%)$.

The bacterial frequencies in the low, medium and high bleeding groups were also evaluated according to the gender and age of the participants. There were no significant differences $(\mathrm{p}<0.05)$ regarding the bacterial frequencies among these groups.

In order to determine the association between $S$. mutans and the periodontal bacteria studied ( $A$. actinomycetemcomitans, C. rectus, $P$. gingivalis, $P$. intermedia and T. forsythia), Pearson's correlation coefficient $(r)$ was calculated, and the results are summarized in Table 2. S. mutans did not show significant association with any periodontal bacteria. Among the periodontal pathogens evaluated, $P$. gingivalis showed the greater correlation coefficient with the presence of $S$. mutans, although with an extremely reduced value $(\mathrm{r}=0.1626)$, which makes this finding irrelevant.

A high percentage of children showed positive samples for $S$. mutans $(71.9 \%)$ and the population presented a mean $\mathrm{dmft} / \mathrm{DMFT}$ value of 6.68 . When the $\mathrm{dmft} / \mathrm{DMTF}$ index was determined for each studied group $(6.96,6.30$ and 6.78 , respectively for low, medium and high bleeding), no significant difference $(\mathrm{p}<0.05)$ was found. In addition, when the mean frequency of $S$. mutans was determined for each studied group $(0.71,0.77$ and 0.63 , re-

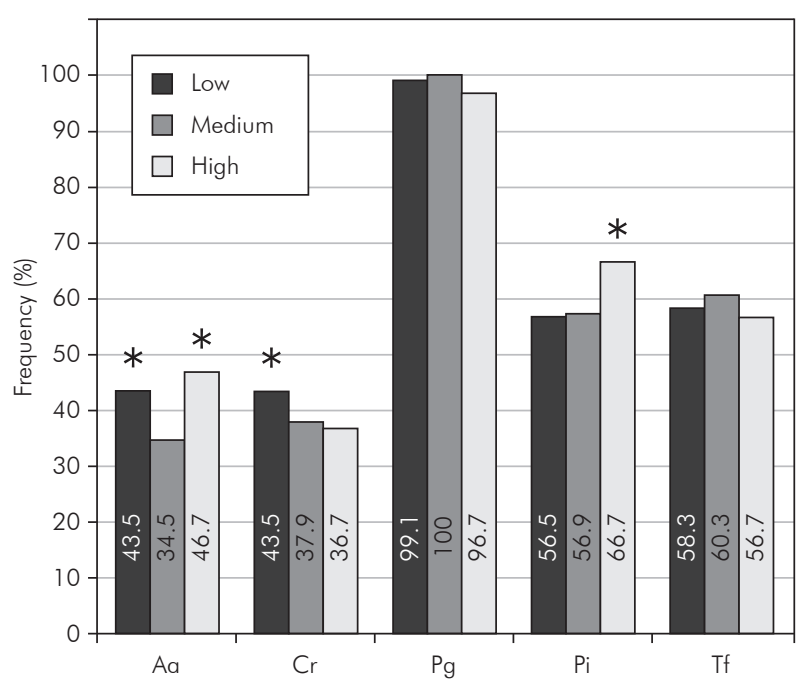

Graph 1 - Detection frequencies (\%) of each bacterium (Aa - A. actinomycetemcomitans, $\mathrm{Cr}-\mathrm{C}$. rectus, $\mathrm{Pg}-\mathrm{P}$. gingivalis, $\mathrm{Pi}-\mathrm{P}$. intermedia and $\mathrm{Tf}-\mathrm{T}$. forsythia) observed in the different groups (Low, Medium and High bleeding). Asterisks indicate statistically significant differences between the groups; ${ }^{*}<0.05$ by the Chi-squared test $\left(X^{2}\right)$.

Table 2 - Pearson's correlation coefficient $(r)$ and $p$ value for S. mutans and the other oral bacteria analyzed (Aa - A. actinomycetemcomitans, $\mathrm{Cr}-\mathrm{C}$. rectus, $\mathrm{Pg}-\mathrm{P}$. gingivalis, $\mathrm{Pi}-\mathrm{P}$. intermedia and $\mathrm{Tf}-\mathrm{T}$. forsythia).

\begin{tabular}{c|c|c|c|c|c}
\hline & Aa & Cr & Pg & Pi & Tf \\
\hline \multirow{3}{*}{ S. mutans } & $r=0.0168$ & $r=0.0104$ & $r=0.1626$ & $r=0.0918$ & $r=-0.0629$ \\
\cline { 2 - 7 } & $p=0.8151$ & $p=0.8854$ & $p=0.0234$ & $p=0.2013$ & $p=0.3814$ \\
\hline
\end{tabular}

Table 3 - DMFT and medium frequency of S. mutans according to the studied group: Low, Medium and High bleeding.

\begin{tabular}{c|c|c|c}
\hline & Low Bleeding (Mean $\pm \mathrm{sd}$ ) & Medium Bleeding (Mean $\pm \mathrm{sd})$ & High Bleeding $($ Mean $\pm \mathrm{sd})$ \\
\hline DMFT & $6.96 \pm 3.78$ & $6.30 \pm 3.89$ & $6.78 \pm 4.27$ \\
\hline S. mutans & $0.71 \pm 0.44$ & $0.77 \pm 0.41$ & $0.63 \pm 0.39$ \\
\hline
\end{tabular}

sd: Standard deviation. 
spectively for low, medium and high bleeding), no significant difference $(\mathrm{p}<0.05)$ was found either. Therefore, no statistical significant association was observed between the presence of $S$. mutans and the $\mathrm{dmft} / \mathrm{DMTF}$ index of each studied group (Table 3).

\section{Discussion}

Periodontal diseases are due to the association between a plaque biofilm and the host responses. A specific group of bacteria, predominantly composed by Gram-negative, anaerobic microorganisms, is implicated in the initiation and progression of periodontal inflammation. The bacterial species $P$. gingivalis, A. actinomycetemcomitans, $P$. intermedia, $T$. forsythia have been identified as good predictors of future clinical attachment loss in susceptible hosts. ${ }^{14}$

In the present study, we showed that marginal biofilm samples from children between 6 and 12 years of age exhibited a high prevalence of oral pathogens. Interestingly, despite the high levels of supragingival biofilm and the high prevalence of periodontopathogens, the majority of the children analyzed $(55.1 \%)$ presented a low bleeding score $(<30 \%)$ (Table 1). On the other hand, the high prevalence of $S$. mutans was compatible with the mean $\mathrm{dmft} / \mathrm{DMTF}$ score (6.68). Considering the ages of the recruited children, between 6 and 12 years, the found DMFT/dmft value (6.68) can be considered high according to the mean DMFT, lower than 1, proposed by the World Health Organization for the age of 12 in the year $2010 .^{10}$

$P$. gingivalis was the pathogen most frequently found $(99 \%)$, followed by T. forsythia $(58.7 \%)$, and P. intermedia (58.2\%). A. actinomycetemcomitans $(41.3 \%)$ and C. rectus $(40.8 \%)$ showed a moderate prevalence. Similar observations were made by McClellan et al. ${ }^{15}$ (1996) who used the same PCR analysis used in the present study, showing that $P$. gingivalis was detected in 40 to $50 \%$ of children ranging from 0 to 2 years old, and in $60 \%$ of teenagers aged 13 to 14 years.

No association between gingival inflammation and the presence of $T$. forsythia and $P$. gingivalis was found in the present study. Similar results were demonstrated by Kisby et al. ${ }^{16}$ (1989) who showed that the prevalences of A. actinomycetemcomitans,
$P$. gingivalis, and $P$. intermedia were approximately the same in healthy and gingivitis children between 8 and 10 years of age.

In accordance with the study of Gafan et al. ${ }^{17}$ (2004), our study also failed to demonstrate any significant difference between healthy and gingivitis children between 5 and 9 years old with respect to the presence of $P$. gingivalis or A. actinomycetemcomitans. Surprisingly, these authors showed that $T$. forsythia was observed more frequently (2.3 times greater) in children without gingivitis compared to individuals with gingivitis. In our study, the presence of A. actinomycetemcomitans was not directly associated with health or disease status. Interestingly, the prevalence of $A$. actinomycetemcomitans was higher in the Low (43.5\%) and High (46.7\%) bleeding groups than in the Medium bleeding group $(34.5 \%)$. Considering all the discrepancies found among the studies in the literature, further future investigations are still required in order to better understand the role played by each one of these cited species in periodontal disease in children.

In addition, our study evaluated the association between the presence of $S$. mutans and the identification of periodontopathogens in the marginal biofilm of children. Interestingly, $S$. mutans did not show significant association with any of the periodontal pathogens analyzed. Previous research has suggested that patients with periodontitis, particularly localized aggressive periodontitis, have minimal tooth decay. ${ }^{18,19}$ Accordingly, a recent study by Fine et al. ${ }^{20}$ (2007) has showed that A. actinomycetemcomitans-positive subjects, who predominantly had localized aggressive periodontitis (LAP), have a salivary factor that significantly reduces the survival of $S$. mutans. This finding suggested an explanation for the fact that the LAP group typically has minimal proximal tooth decay. The lack of any kind of association between the prevalence of $S$. mutans and the periodontopathogens in our study may be due to the high prevalence of plaque (plaque index greater than $80 \%$ ) manifested by the population of children studied.

Our study is in accordance with a previous study by Tanner et al. ${ }^{21}$ (1989) who demonstrated that putative periodontal pathogens colonization establish- 
es in early years of life, therefore pointing out the need for more effective preventive dental programs.

\section{Conclusion}

It may be concluded that Brazilian children, ranging from 6 to 12 years of age, with high levels of supragingival biofilm, presented a high prevalence of periodontopathogens and $S$. mutans. It may also be concluded that in spite of the high levels of supragingival biofilm and high prevalence of periodon-

\section{References}

1. Darveau RP, Tanner A, Page R. The microbial challenge in periodontitis. Periodontology 2000. 1997;14(3):12-32.

2. Baelum V, Fejerskov O, Karring T. Oral hygiene, gingivitis and periodontal breakdown in adult Tanzanians. J Periodontal Res. 1986;21(3):221-32.

3. Baelum V, Fejerskov O, Manji F. Periodontal diseases in adult Kenyans. J Clin Periodontol. 1988;15(7):445-52.

4. Löe H, Anerud A, Boysen H. The natural history of periodontal disease in man: prevalence, severity, and extent of gingival recession. J Periodontol. 1992;63(6):489-95.

5. Lovegrove JM. Dental plaque revisited: bacteria associated with periodontal disease. J N Z Soc Periodontol. 2004;(87):721.

6. Loesche WJ. Role of Streptococcus mutans in human dental decay. Microbiol Rev. 1986;50:353-80.

7. Thenisch NL, Bachmann LM, Imfeld T, Leisebach Minder $\mathrm{T}$, Steurer J. Are mutans streptococci detected in preschool children a reliable predictive factor for dental caries risk? A systematic review. Caries Res. 2006;40(5):366-74.

8. Butler BL, Morijon O, Low SB. An accurate, time-efficient method to assess plaque accumulation. J Am Dent Assoc. 1996;127(12):1763-6.

9. Ainamo J, Bay I. Problems and proposals for recording gingivitis and plaque. Int Dent J. 1975;25(4):229-35.

10. World Health Organization. Oral Health Surveys: Basic Methods. $4^{\text {th }}$ ed. Geneva; Swiss; 1997.

11. Cortelli JR, Aquino D, Cortelli SC, Fernandes CB, Carvalho-Filho J, Franco GCN et al. Etiological analysis of initial colonization of periodontal pathogens in oral cavity. J Clin Microbiol. 2008;46(4):1322-9. topathogens found in Brazilian children, ranging 6 to 12 years of age, the majority of them presented only mild inflammation revealed by a low bleeding score. On the other hand, high levels of $S$. mutans were compatible with poor dental status, i.e., high mean $\mathrm{dmft} / \mathrm{DMTF}$ scores. Our study indicated that whereas $P$. intermedia was more associated with inflamed tissues, C. rectus was more associated with periodontal health.

12. Igarashi T, Yamamoto A, Goto N. Direct detection of Streptococcus mutans in human dental plaque by polymerase chain reaction. Oral Microbiol Immunol. 1996;11(5):294-8.

13. Querido SMR, Cortelli SC, Araujo MWB, Cortelli JR. Clinical and microbial evaluation of dental scaling associated with subgingival minocycline in chronic periodontitis subjects. Braz Oral Res. 2004;18(2):110-5.

14. Socransky SS, Haffajee AD, Smith C, Dibart S. Relation of counts of microbial species to clinical status at the sampled sites. J Clin Periodontol. 1991;18(10):766-75.

15. McClellan DL, Griffen AL, Leys EJ. Age and prevalence of Porphyromonas gingivalis in children. J Clin Microbiol. 1996;34(8):2017-9.

16. Kisby LE, Savitt ED, French CK, Peros WJ. DNA probe detection of key periodontal pathogens in juveniles. J Pedod. 1989;13(3):222-9.

17. Gafan GP, Lucas VS, Roberts GJ, Petrie A, Wilson M, Spratt DA. Prevalence of periodontal pathogens in dental plaque of children. J Clin Microbiol. 2004;42(9):4141-6.

18. Albandar JM, Brown LJ, Löe H. Dental caries and tooth loss in adolescents with early-onset periodontitis. J Periodontol. 1996;67(10):960-7.

19. Sioson PB, Furgang D, Steinberg LM, Fine DH. Proximal caries in juvenile periodontitis patients. J Periodontol. 2000;71(5):710-6.

20. Fine DH, Furgang D, Goldman D. Saliva from subjects harboring Actinobacillus actinomycetemcomitans kills Streptococcus mutans in vitro. J Periodontol. 2007;78(3):518-26.

21. Tanner A, Bouldin HD, Maiden MF. Newly delineated periodontal pathogens with special reference to selenomonas species. Infection. 1989;17(3):182-7. 